\title{
MIHOP: Energy Efficient Scheme in Wireless Sensor Network
}

\author{
Nisha Patil ${ }^{1}$, Shivom Tiwari ${ }^{2}$ \\ ${ }^{I}$ (ME Student, Department of EXTC, Siddhant College of Engineering, Pune, India) \\ ${ }^{2}$ (Assistant Professor, Department, of EXTC, Siddhant College of Engineering, Pune, India)
}

\begin{abstract}
Wireless sensor networks (WSNs) have emerged as an effective solution for a wide range of applications. Most of the traditional WSN architectures consist of static nodes which are densely deployed over a sensing area. Various radio applications in the wireless sensor networks consist of nodes which operate on batteries, so that energy consumption must be minimized while satisfying given throughput and delay requirements. It is critical to prolong the network lifetime by minimizing the energy consumption of each node. In this paper, various energy efficient schemes are analyzed. Also new method which gives improved performance than existing schemes is proposed called MIHOP technique. The MIHOP (MIMO and Multi-hop) method combines cluster-based virtual MIMO and multi-hop technologies. The multi hop mode is employed in transmitting data when the related sensors are located within a specific number of hops from the sink, and the virtual MIMO mode is used in transmitting data from the remaining sensor nodes. A controllable mobile sink that reduces the energy consumed in sensor transmission is also adopted for data collection.. The theoretical analysis shows that the MIHOP scheme significantly outperforms individual virtual MIMO, multi-hop schemes in terms of energy efficiency when energy consumption in transmission and circuitry are considered.
\end{abstract}

Keywords: - Energy efficiency, Mobile sink, multi-hop network, MIHOP, virtual MIMO, Wireless sensor network.

\section{INTRODUCTION}

The problem of data collection in sparse sensor networks is encountered in many scenarios. The objective is to collect data from sensors and deliver it to an access point in the infrastructure. These systems are expected to run unattended for long periods of time (order of months). The principal constraint is the energy budget of the sensors which is limited due to their size and cost. One of the major energy expenditures is in communicating the sensor readings, in raw or processed form, from the sensors to a central user location. Usually, these readings are relayed to a base station using ad hoc multi hop routes in the sensor network. A problem with this approach, however, is that the nodes closer to the base station relay data from nodes that are farther away. Thus, the nodes closest to the base station consume batteries faster than the remaining network, leading to a non uniform depletion of energy in the network. Once the nodes with connectivity with the base station exhaust their energy, the network is disconnected and, hence, considered dead for all practical purposes.

A significant advantage in network lifetime can be gained if the energy spent in relaying data can be saved. An alternative for data transfer that does not involve relaying it over multiple hops is to use mobility. A mobile node moving through the network deployment region can collect data from the static sensor nodes over a single hop radio link as and when the mobile node is within radio range of the static nodes. This naturally avoids multi hop relaying and reduces the energy overhead at nodes near the base station, enabling the network to last longer. This may increase the latency of data transfer, but is acceptable in several delay-tolerant applications, such as in environmental studies, city traffic monitoring application, habitat monitoring scenario. In certain applications, mobile elements already exist in the deployment environment and a network node can be attached to these mobile elements for data collection Otherwise, mobile nodes can be added to the system for data collection. In this case, the mobile node is a part of the network infrastructure itself and can be controlled by the network as required.

Hence the scheme, called MIHOP (MIMO and Multi-hop) is used which combines the advantages of multi-hop and STBC based virtual MIMO technologies. In MIHOP, a mobile sink is managed so that it moves along a prepared path and pauses at certain locations to broadcast routing information. The sensor nodes near the sink are located within a specific predefined number of hops, and constitute a multi-hop network. Each node transmits data to the sink hop by hop. The sensor nodes further from the sink may use STBC-based virtual MIMO technology to transmit data \& handle sparse or disconnected networks. Disconnected networks may be desirable in certain applications due to the following reasons: First, the placement of sensor nodes (dictated by the requirements of field experts) may lead to disconnected networks. Second, even to sample the whole area of deployment, the sampled physical phenomenon may have a low spatial variation, i.e., it may be sufficient to place sensors at distances which are larger than the available communication range in the environment. 


\section{RELATED WORK}

Energy-constrained networks, such as wireless sensor networks, have nodes that are typically powered by batteries, for which replacement or recharging is very difficult. Finite energy can only support the transmission of a finite amount of information. Therefore, minimizing the energy consumption for information transmission becomes one of the most important design considerations for such networks. A certain amount of research has recently been done to investigate various Energy efficient transmission schemes.

In [1] Yong Yuan et al. proposed a novel multi hop virtual multiple-input multiple-output (MIMO) communication protocol by the cross-layer design to jointly improve the energy efficiency, reliability, and endto-end (ETE) QoS provisioning in wireless sensor network (WSN). In the protocol, the traditional low-energy adaptive clustering hierarchy protocol is extended by incorporating the cooperative MIMO communication, multi hop routing, and hop-by-hop recovery schemes. Based on the protocol, the overall energy consumption per packet transmission is modeled and the optimal set of transmission parameters is found.

In [2] CUI Shuguang, et.al proposed MIMO systems based on Alamouti diversity schemes, which have good spectral efficiency but also more circuitry that consumes energy. They extend this energy-efficiency analysis of MIMO systems to individual single-antenna nodes that cooperate to form multiple antenna transmitters or receivers. In this, the authors have used a cooperative transmission scheme based on distributed space-time block coding and conduct a systematic analysis on the resulting energy consumption.

In [3] Yi Gai, Lin Zhang et al. proposed an energy model for wireless sensor networks based on the cooperative MIMO (multiple-in-multiple-out) technique, taking into consideration of both the transmission and data aggregation energy. In [4] SUSHANT JAIN et al. analyze an architecture based on mobility to address the problem of energy efficient data collection in a sensor network. Their approach exploits mobile nodes present in the sensor field as forwarding agents. As a mobile node moves in close proximity to sensors, data is transferred to the mobile node for later depositing at the destination. Energy conservation in Wireless Sensor Networks (WSNs) has always been a crucial issue and has received increased attention in the recent years. Numerous techniques, such as multi-hop and virtual MIMO, as well as mobile data gathering schemes have been developed for this purpose.

\subsection{MIMO \& Cooperative MIMO}

Multiple-input-multiple-output (MIMO), or multiple antenna, communication is one of the techniques that has gained considerable importance in wireless systems during recent years. However, a drawback of MIMO techniques is that they could require complex transceiver circuitry and large amount of signal processing power that may lead to large power consumptions at the circuit level. Thus, in evaluating the applicability of MIMO techniques to energy-limited wireless sensor networks, one should take into account the circuit power consumption as well as the transmit power consumption. In virtual MIMO-based cooperative communications architecture for energy-limited wireless sensor networks. Virtual multiple transmit antenna arrays are created out of single antenna sensor nodes via local transmissions. The sensor nodes in a wireless sensor network can be of small dimensions. Thus, it may not be realistic for these sensor nodes to have multiple antennas. However, it is possible to implement a virtual MIMO communication architecture in such energy-limited, distributed wireless sensor networks via sensor cooperation, as reported in [10].

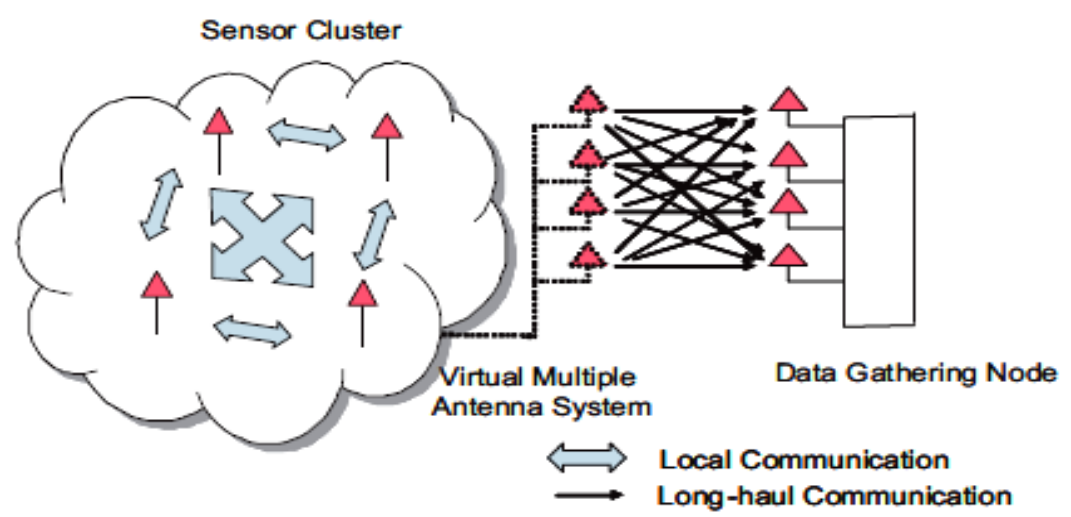

Fig.1 A virtual MIMO communication-based wireless sensor network

A common scenario in distributed wireless sensor networks is that of a lead-sensor and a set of data collection nodes (Fig. 1). In such a wireless sensor network model, the proposed virtual MIMO-based communications can be achieved as follows: Suppose a set of data collection nodes have data to be sent to the data gathering node (DGN). Each of these sensors which are assumed to be close to each other broadcasts their 
data to the others in the set using a time-division multiple-access scheme. This step is known as the local communications at the transmitter side. At the end of this step each of the data collection nodes have data from all the sensor nodes. This enables space-time block coding assuming each data collection node corresponds to a distinct transmit antenna element in a centralized multiple transmit antenna system. Once the space-time coding is performed each sensor node transmits the space-time code symbols corresponding to a specific transmit antenna element to the DGN. This step is known as the longhaul communications. The DGN is assumed to be different from the low-end data collection nodes. First, it does not have any energy constraints attached to it (or compared to the data collection nodes, the DGN has much longer battery life). Second, this sensor can be of larger physical dimensions thus enabling it to have multiple receiver antenna capability. This allows realization of true MIMO capability with only the transmitter side local communications.

\subsection{Multihop Routing}

Multi-hop routing and direct transmission are conventional transmission schemes for WSNs. In multihop networks, encoded data are generated and transmitted by one sensor to an intermediate node, and then relayed to a sink hop by hop. Ref. [5] shows that multi-hop transmission is more energy-efficient than singlehop transmission in general WSNs. However, when a sink is far from the sensor area or the area is so large that most sensor nodes need numerous hops to reach the sink, considerable retransmitting energy is consumed during transmission, thereby significantly accelerating node depletion. To overcome above system drawbacks MIHOP System model is proposed.

\subsection{MIHOP System Model}

\section{PROPOSED SCHEME}

The MIHOP (MIMO and Multi-hop) scheme combines cluster-based virtual MIMO and multi-hop technologies. The multihop mode is employed in transmitting data when the related sensors are located within a specific number of hops from the sink, and the virtual MIMO mode is used in transmitting data from the remaining sensor nodes. Figure 2 illustrates the system model of MIHOP. The sink works similar to a base station that broadcasts and gathers information. In the multi-hop network formation stage, a sink broadcasts routing information packets, and the sensors that receive the packets function as first-hop nodes. These nodes rebroadcast the routing information packets in wireless channels, and the entire multi-hop network is created through the hop-by-hop routing of an algorithm. The range of a multi-hop network is limited by maximum number of hops $M_{H}$, which can be optimized according to the model proposed in further section. This optimization minimizes energy consumption. Every two nodes with hops greater than $M_{H}$ form a cluster on the basis of minimum cooperation range [5].

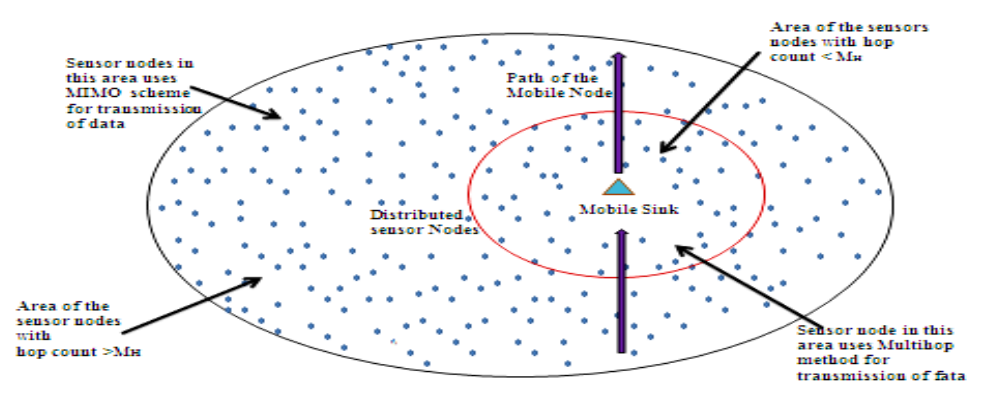

Fig.2 System model of MIHOP

The STBC-based virtual MIMO scheme proposed which is adopted for data transmission to a sink. The nodes in one cluster are assumed located on the same tier of the virtual MIMO network, and the distance between two adjacent tiers is denoted as $r$ (Figure 1). Two nodes in one cluster should be located on the $k^{\text {th }}$ tier and the collaboration distance between these nodes is expressed as $d$. The transmission distance from node to sink is denoted as $k_{r}$. Remaining single nodes can transmit data to a sink by SISO technology alone. After each sensor selects a transmission mode, the TDMA schedule is used by the sink to determine the sequence at which the sensors transmit data. Design flow for MIHOP technique is shown below, 


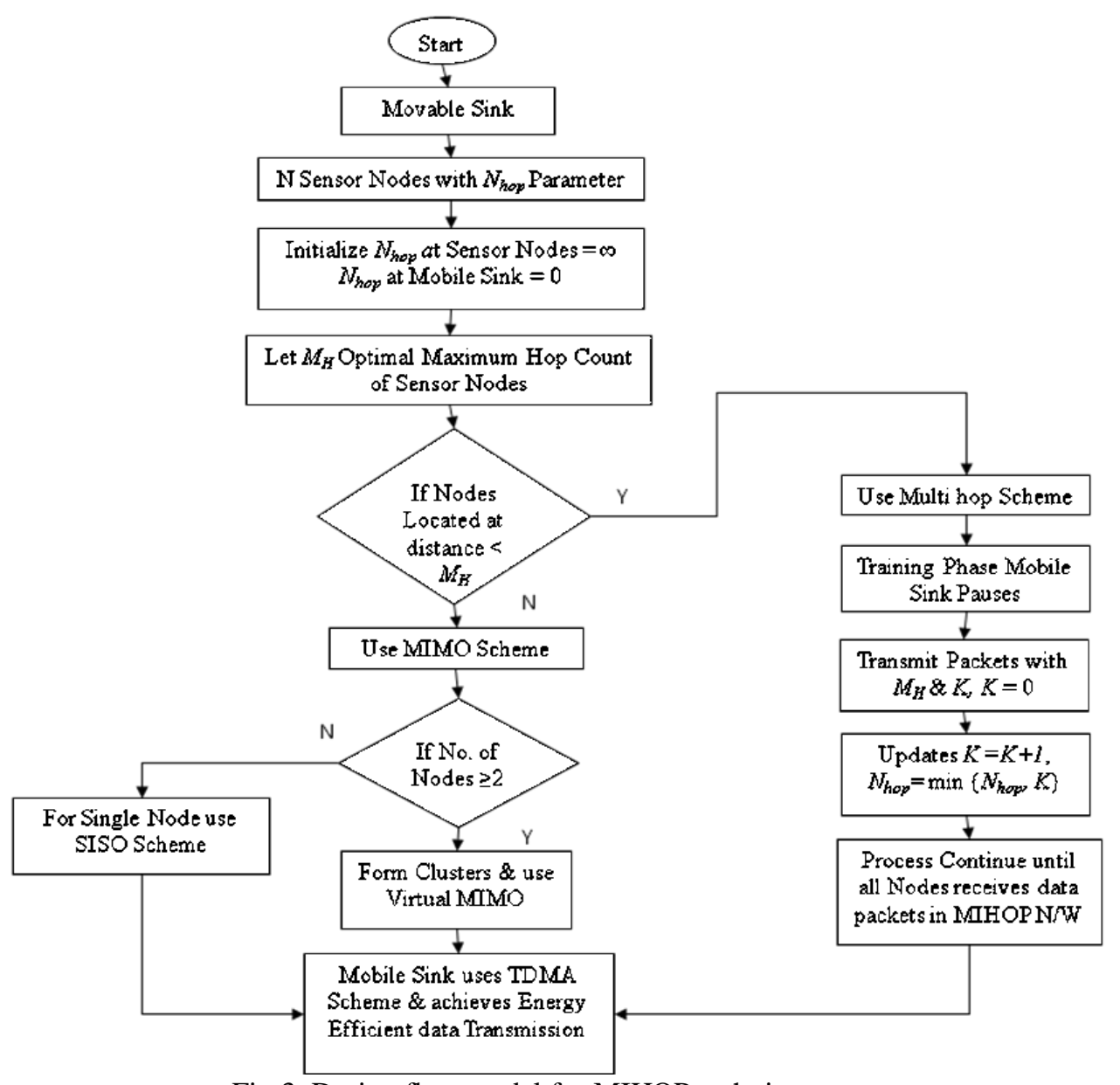

Fig.3. Design flow model for MIHOP technique

\subsection{Mobile Sink:}

Suppose $\mathrm{N}$ sensor nodes are distributed in the sensing area as shown in Figure 4, to which a mobile sink is introduced. Adaptive Motion Control algorithm is used to control the motion of the mobile node [7]. This sink is employed to enable movement along a fixed path, which is a cross path. It pauses at certain data gathering points to broadcast BEACON packets and periodically collect data from sensor nodes. The sink is equipped with two antennas, and each sensor has a single antenna for uploading data. Every packet has a fixed length of $L$ bits. The network layer algorithms are used to select a route from the sink to each sensor in the multi-hop network.

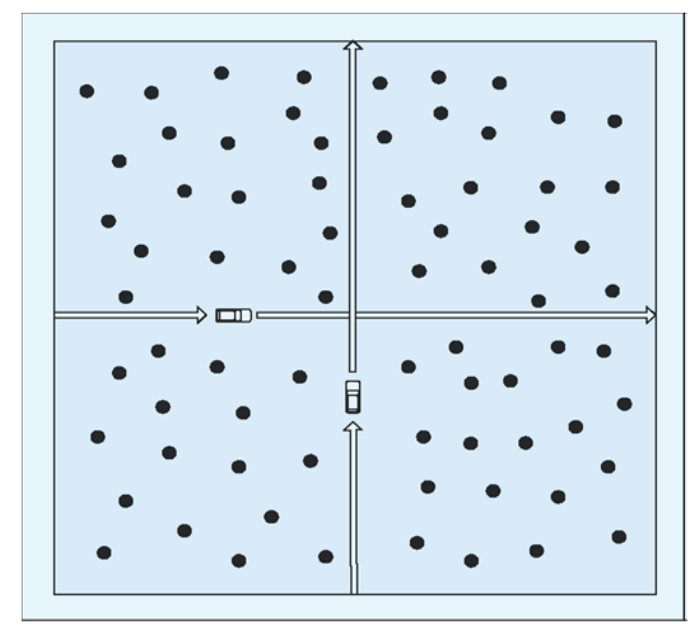

Fig.4 Movement of the mobile sink 
Each sensor node maintains a parameter $N_{\text {hop }}$, which represents the shortest hop count to the mobile sink. The value of $N_{h o p}$ is initialized as infinite, but the $N_{h o p}$ on the mobile sink is set as 0 . In the training phase, the mobile sink stops at a data gathering point and broadcasts BEACON packets with $M H$ and $K$. $K$ is initialized as 0 . Each sensor node receiving the BEACON packet adds 1 to $K$, then updates its $N_{\text {hop }}$ into $N_{\text {hop }}=\min \left\{N_{\text {hop }}\right.$, $K\}$ and rebroadcasts the BEACON with the new $K$. This process continues until all the nodes in the network receive a BEACON hop by hop. The mobile sink is then moves onto the next point and again broadcast routing information. After the training phase, sensor nodes with $N_{h o p}$ higher than $M_{H}$ form into clusters and the virtual MIMO mechanism is used to transmit data to the mobile sink. Other nodes with $N_{\text {hop }}$ lower or equal to $M_{H}$ adopt multi-hop transmission technology. Also energy consumption can be reduced by selecting specific path of mobile sink node in wireless sensor network. It has been observed that cross path or X-path shows minimum power consumption than rectangular path \& zigzag path. Different paths are shown below,

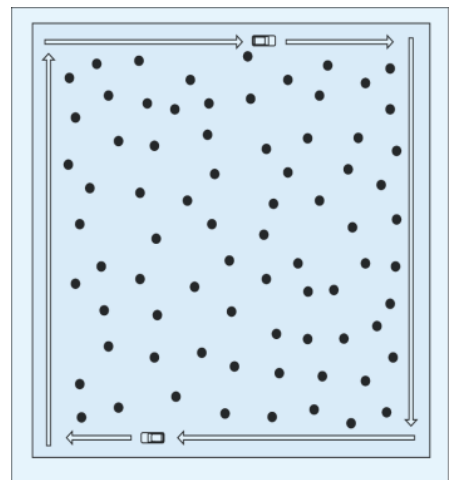

Fig.5 Rectangular path

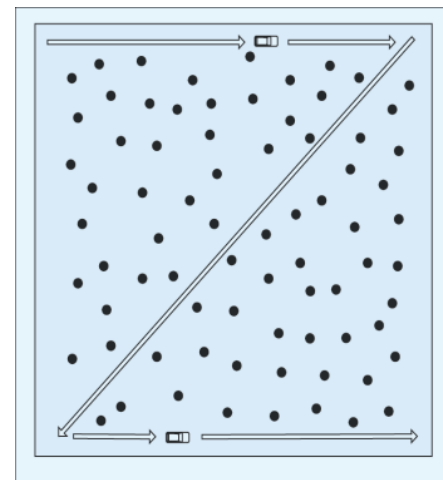

Fig.6 Zigzag path

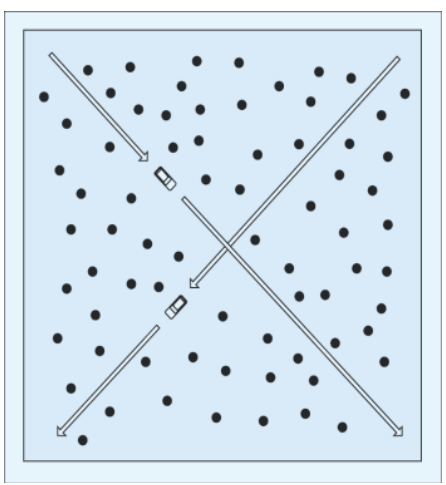

Fig.7 X path

\section{ENERGY CONSUMPTION ANALYSIS}

Energy consumption models of virtual MIMO, multi-hop technology and SISO methods are derived. The method for determining the optimal number of hops is provided, and the energy consumption model of MIHOP is constructed.

The total energy consumption $E_{M I H O P-t o t a l}$ of the MIHOP scheme comprises three components: the energy consumption levels of the multi-hop network, virtual MIMO scheme, and SISO scheme. $N_{M 1}, N_{M 2}$, and $N_{M 3}$ are assumed to be the number of sensor nodes in the multi-hop network, virtual MIMO scheme, and SISO scheme, respectively. $N_{\text {total }}$ denotes the total number of nodes in the MIHOP scheme, where $N_{\text {total }}=N_{M 1}+N_{M 2}+N_{M}$. If $n_{i}$ indicates the $i^{\text {th }}$ node in different transmission modes, its energy consumption $E_{i}$ can be expressed as,

$$
E^{i}= \begin{cases}E_{\text {Muitihop }}^{i} & \text { When } n_{i} \text { is in the multi }- \text { hop network } \\ E_{\text {MIMo }}^{i} & \text { When } n_{i} \text { is in the virtual MIMO scheme } \\ E_{\text {SISO }}^{i} & \text { When } n_{i} \text { is in the SISO scheme }\end{cases}
$$

Thus, $E_{\text {MIHOP-total }}$ can be expressed as follows,

Where, $E_{\text {Multihop }}^{i}=E_{\text {Multihop }}^{i}\left(M, d_{\text {hop }}\right) \quad\left(1 \leq M \leq M_{H}\right)$

$$
E_{\text {MIHOP_total }}=\sum_{i=1}^{N_{\text {total }}} E^{i}=\sum_{i=1}^{N_{M_{1}}} E_{\text {Mulitihop }}^{i}+\sum_{i=1}^{N_{M_{2}}} E_{\text {MIMO }}^{i}+\sum_{i=1}^{N_{M_{s}}} E_{\text {SISO }}^{i}
$$

\subsection{Algorithm for determining the optimal hop count in multi-hop networks:}

Existing methods indicate that multi-hop networks are advantageous for data transmission in small areas, whereas virtual MIMO schemes are more energy efficient for large-area transmission. The MIHOP scheme determines the maximum allowable transmission distance (in a multi-hop network) at which the energy efficiency of multi-hop transmission can be maintained. Within this distance, the multi hop networks outperform virtual MIMO schemes; beyond this distance, virtual MIMO technologies exhibit better performance than do the former. As shown in Figure 1, the problem lies in identifying the optimal maximum hop count in the multi-hop network given that the transmission distance from the node in tier $n$ to the sink is approximately estimated as $n \times$ $r$.

The algorithm for determining $M_{H}$ is described as follows,

1. Initialize $M_{H}=1 ; r$ is a fixed value

2. While TRUE

3. If $E_{\text {Multihop }}^{i}\left(M_{H}, r\right)<E_{\text {MIMO }}^{i}\left(M_{H} \times r\right)$

4. $M_{H}{ }^{++}$ 

5. else
6. break;
7. end while.

In this algorithm $E_{\text {MIMO }}^{i}$ is the energy consumption of the $i^{\text {th }}$ node that belongs to the $j^{\text {th }}$ cluster in the virtual MIMO scheme. As an extension of Eq. (10), $E_{\text {MIMO }}^{i}$ can be expressed as:

$E_{\text {MIMO }}^{i}=E_{\text {MIMO c }}^{j} / 2$

In the algorithm, the comparison of one node energy consumption between two transmission schemes is shown in line 3. $M_{H}$ progressively increases and stabilizes at the value at which the comparison is mismatched. The final value of $M_{H}$ is the optimal hop count in the MIHOP scheme. Thus using Mobile Sink node along with MIHOP technique more amount of sensor's energy can be saved.

\section{CONCLUSIONS}

An energy-efficient transmission scheme for mobile data gathering in WSNs is proposed. The motivation for doing this was to save energy in the embedded sensor nodes and increase the useful service time of a deployed system. The MIHOP technique combines cluster-based virtual MIMO and multi-hop technologies along with mobile sink node. The key intuition was that using a mobile node to establish shorter data routes reduces the data relaying overhead, especially at the nodes close to the data egress point in the network. The sensor nodes within two hops operate in multi-hop mode as they transmit data, and the remaining nodes operate in virtual MIMO of SISO mode. An algorithm for determining the optimal number of hops required to form a multi-hop network is derived, and the energy consumption model of MIHOP is developed. The MIHOP scheme significantly outperforms individual virtual MIMO, multi-hop technologies and double string network in terms of energy efficiency.

\section{REFERENCES}

[1] Yong Yuan, Zhihai He, and Min Chen "Virtual MIMO-Based Cross-Layer Design for Wireless Sensor Networks" 2006, IEEE.

[2] CUI Shuguang, GOLDSMITH A J, BAHAI A. "Energy Efficiency of MIMO and Cooperative MIMO Techniques in Sensor Networks' [J]. IEEE Journal on Selected Areas in Communications, 2004, 22(6): 1089-1098.

[3] Yi Gai, Lin Zhang and Xiuming Shan "Energy Efficiency of Cooperative MIMO with Data Aggregation in Wireless Sensor Networks",

[4] SUSHANT JAIN, RAHUL C. SHAH et al. "Exploiting Mobility for Energy Efficient Data Collection in Wireless Sensor Networks". Mobile Networks and Applications 11, 327-339, 2006.

[5] TANG Qiuling, SUN Changyin, WEN Huan, et al. "Cross-Layer Energy Efficiency Analysis and Optimization in WSN"'[C]// Proceedings of the International Conference on Networking, Sensing and Control: April 10-12, 2010. Chicago, USA, 2010: 138-142.

[6] LIU Danpu, ZHANG Kailin, DING Jie "Energy-Efficient Transmission Scheme for Mobile Data Gathering in Wireless Sensor Networks” 2013, China Publication.

[7] ZHOU Zhong, ZHOU Shengli, CUI Shuguang et al. "Energy-Efficient Cooperative Communication in Cluster Wireless Sensor Network "[J] IEEE Transactions on Vehicular Technology 2008, 57(6): 36183628.

[8] SOMASUNDARA A.A., KANSAL A, JEA D. D., et al. Controllably Mobile Infrastructure for Low Energy Embedded Networks [J]. IEEE Transaction on Mobile Computing, 2006, 5(8): 958973.

[9] Tracy Camp, Jeff Boleng, Vanessa Davies "A Survey of Mobility Models for Ad Hoc Network Research"2002 Wireless Communication \& Mobile Computing (WCMC).

[10] A. Chakrabarti, A. Sabharwal, and B. Aazhang, "Using Predictable Observer Mobility for Power Efficient Design of Sensor Networks," Proc. Second Int'l Workshop Information Processing in Sensor Networks (IPSN), 2003.

[11] DING Jie, LIU Danpu, WU Huari. "A Cooperative MIMO Transmission Scheme for Cluster based Wireless Sensor Networks [J]”. China Communications, 2010, 7(5): 14-22.

[12] AKSU A, ERCETIN O. "Reliable Multi-Hop Routing with Cooperative Transmissions in Energy Constrained Networks [J]". IEEE Transaction on Wireless Communications, 2008, 7(8): 2861-2865.

[13] CUI Shuguang, GOLDSMITH A J. "Energy Efficient Routing Based on Cooperative MIMO Techniques" [C]//Proceedings of the IEEE International Conference on Acoustics, Speech, and Signal Processing: March 18-23, 2005. Philadelphia, USA, 2005: 805-808.I

[14] JAYAWEERA S K. "Energy Analysis of MIMO Techniques in Wireless Sensor Networks"[C]// Proceedings of the 38th Annual Conference on Information Sciences and Systems: March 17-19, 2004. Princeton, USA. 